\title{
Magnetoresistance studies of metallic granular and ceramic thin films for microsensor applications
}

\begin{abstract}
Thin films of granular metal and manganites ceramic were fabricated using RF magnetron sputtering and pulsed laser deposition techniques. The GMR effect is highly influenced by the shape, size and distribution of the magnetic entities. The highest GMR value of $7.6 \%$ for Ag87.0Fe9.5Co3.5 granular film measured at 1 Tesla at room temperature had been obtained. In the polycrystalline manganites system, low-field magnetoresistance (LFMR) had been observed due to the grain boundaries. LFMR of $4.3 \%$ and $6.2 \%$ measured at 0.1 Tesla at room temperature have been observed for both $\mathrm{La}-\mathrm{Sr}-\mathrm{Mn}-\mathrm{O}$ and $\mathrm{La}-\mathrm{Ba}-\mathrm{Mn}-\mathrm{O}$ samples. For the La-Sr-Mn-O film, LFMR ratio of $4.5 \%$ has been observed. This value meets the requirement as sensor element and it is currently used in many applications such as head sensing in hard disk.
\end{abstract}

Keyword: Gaint Magnetoresistance (GMR); Colossal Magnetoresistance (CMR); Magnetoresistance (MR); Granular metallic film; Ceramic manganites 